Introduction The implantation of Flow-Diverter stents (FD) is an effective technic for the treatment of intracranial aneurysms but can expose to severe hemorrhagic and/or ischemic complications due to their metallic structure. CD31 is a trans-membranous protein highly expressed on the luminal surface of arteries and endowed with a contact-driven attractive effect on endothelial cells, and an inhibitory effect of platelets and leucocytes activation. The goal of this study was to evaluate, in vitro and in vivo, whether a coating with P8RI, a biomimetic peptide of CD31, could improve the biocompatibility of FD.

Methods The coating of metal pellets and Silk Vista Baby ${ }^{\circledR}$ (Balt, France) FDs with P8RI was obtained via a series of dipcoating steps, including the formation of an intermediate polydopamine (PDA) layer. In vitro, the adhesion of endothelial cells under different conditions was tested on uncoated and coated metal pellets (PDA alone and PDA with P8RI), and their thrombogenic and inflammatory phenotype were monitored. In vivo, we used a validated elastase-induced saccular carotid aneurysm model in rabbits, separated into three groups: a test group with P8RI-coated FD (P8RI), and two control groups with unmodified FD (UFD) and PDA-coated FD (PDA). Angiographic results were evaluated at 1 and 3 months. Histological, scanning electron and multiphoton microscopy analyses were assessed at 1 month. Patency of covered branches was also evaluated on FD placed in the abdominal aorta covering lumbar arteries.

Results In vitro, P8RI coating promotes adhesion of endothelial cells and induces a less inflammatory and less thrombogenic endothelial cell phenotype. In vivo, 25 aneurysms were created in 25 rabbits and were treated with 7 UFD, 9 PDA and 9 P8RI FDs. There was no significant difference in complete occlusion rate. Histological and microscopy analyses at 1 month showed that the coating with the P8RI peptide improved the integration of the device at the blood vessel interface and the quality of its endothelialization (figure 1). All covered arteries remained patent with no stenosis in all 3 groups.

Conclusion P8RI coating of FD improves biocompatibility and healing process of aneurysm treatment. These results are a crucial step towards a translation to clinical, this technology could be extended also to other intra-arterial devices used in interventional neuroradiology.

Disclosures J. Cortese: 1; C; Fondation pour la Recherche Médicale (DEA20170637766). C. Rasser: None. G. Even: None. C. Choqueux: None. S. Bardet: None. J. Caroff: None. L. Spelle: None. G. Caligiuri: None. A. Rouchaud: None.

\section{0-014 DOUBLE STENT-ASSISTED COILING OF INTRACRANIAL ANEURYSMS WITH THE NEUROFORM ATLAS STENT IN $Y$ AND X CONFIGURATIONS: IMMEDIATE AND MIDTERM ANGIOGRAPHIC AND CLINICAL FOLLOW-UP}

G Ciccio ${ }^{*}$, T Robert, R Fahed, S Smajda, R Blanc, M Piotin. NRI, Fondation Rothschild, Paris, France

\subsection{6/neurintsurg-2019-SNIS.14}

Object Self-expandable stents have broadened the spectrum of endovascular treatment of intracranial aneurysms. However procedures involving double stenting in $\mathrm{Y}$ or $\mathrm{X}$ configuration carry a relatively high risk of procedural complications. The Neuroform ATLAS, the evolution of Neuroform EZ, is a nitinol self-expanding hybrid/open-cell stent, which can be delivered through a low-profile 0.017 inch catheter. We present our experience in the treatment of intracranial aneurysms with this stent in $\mathrm{Y}$ and $\mathrm{X}$ configuration.

Methods We prospectively maintained a database from consecutive patients who underwent double stent-assisted coiling with Neuroform ATLAS from July 2015 to February 2019. Clinical and angiographic results were analyzed.

Results Fifty-six patients harboring 56 aneurysms were treated with double stenting: 53 ' $\mathrm{Y}$ ' configurations, 3 ' $\mathrm{X}$ ' configurations. Deployment was successful in all but one case of $\mathrm{Y}$ stenting, which was prematurely interrupted because of aneurysm perforation. Post-treatment control angiography showed complete occlusion in 33 cases (60\%), neck remnant in 8 cases $(14.5 \%)$ and incomplete occlusion in 14 cases $(25.4 \%)$. The overall symptomatic peri-procedural complication rate was $14 \%$. The overall morbidity rate was $7.1 \%$. Thirty-seven aneurysms underwent follow-up (66\%, mean duration: 16 months): 32 aneurysms (86.4\%) were completely occluded, 3 aneurysms (8.1\%) had a neck remnant, and 2 aneurysms (5.4\%) were incompletely occluded.

Conclusion The Neuroform ATLAS is an effective device for treatment of complex intracranial aneurysms, allowing good conformability, high level of navigability and easy mesh crossing to perform $\mathrm{Y}$ or $\mathrm{X}$ stenting procedures. The rate of procedural complications remains non negligible, and indication of double-stenting procedure should always be discussed in a multidisciplinary meeting.

Disclosures G. Ciccio': None. T. Robert: None. R. Fahed: None. S. Smajda: None. R. Blanc: None. M. Piotin: None.

\section{0-015 COMPARISON OF IMAGE QUALITY OF LIQUID EMBOLIC AGENTS}

J Mason*. Radiology, Baylor College of Medicine, Houston, TX

\subsection{6/neurintsurg-2019-SNIS.15}

Introduction Liquid embolic agents (LEAs) play a major role in the treatment of cerebral arteriovenous malformations (AVMs) and dural arteriovenous fistulas (DAVFs). Injection under subtracted fluoroscopy (Blank Road Map, RM) is the preferred technique, especially when using ethylene-vinylcopolymer based agents. Optimal visual control during injection is crucially important to avoid catheter entrapment or non-target embolization and is strongly dependent on Road Map (RM) quality. Available LEAs differ in their radiopacity the main factor for visual control. We present a comparison study of radiographic visibility of various LEAs using a novel injectable angiographic phantom.

Methods An injectable angiographic phantom was designed with parallel tubings between 313 and 1000 micron. Under $\mathrm{RM}$, eight radiopaque liquid agents were injected: Onyx 18,34

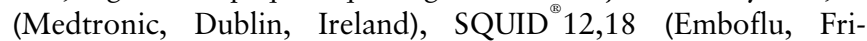
bourg, Switzerland), PHIL 25,30 (MicroVention, Tustin, CA, USA), Trufill ${ }^{\circledast}$ (NBCA) (Cordis Neurovascular, Miami, FL, USA) $30 \%$ dilution and Omnipaque 300 (GE Healthcare, Chicago, IL, USA). The phantom was imaged using an Artis Zeego system (Siemens Healthineers, Erlangen, Germany) with consistent settings ('RM Glue', RM K40 EA3, 15 p/s). Image analysis was performed with ImageJ (NIH, Bethesda, Maryland) and Matlab (MathWorks, Inc., Natick, MA). Contrast resolution (CR) was evaluated as a contrast to noise ratio 

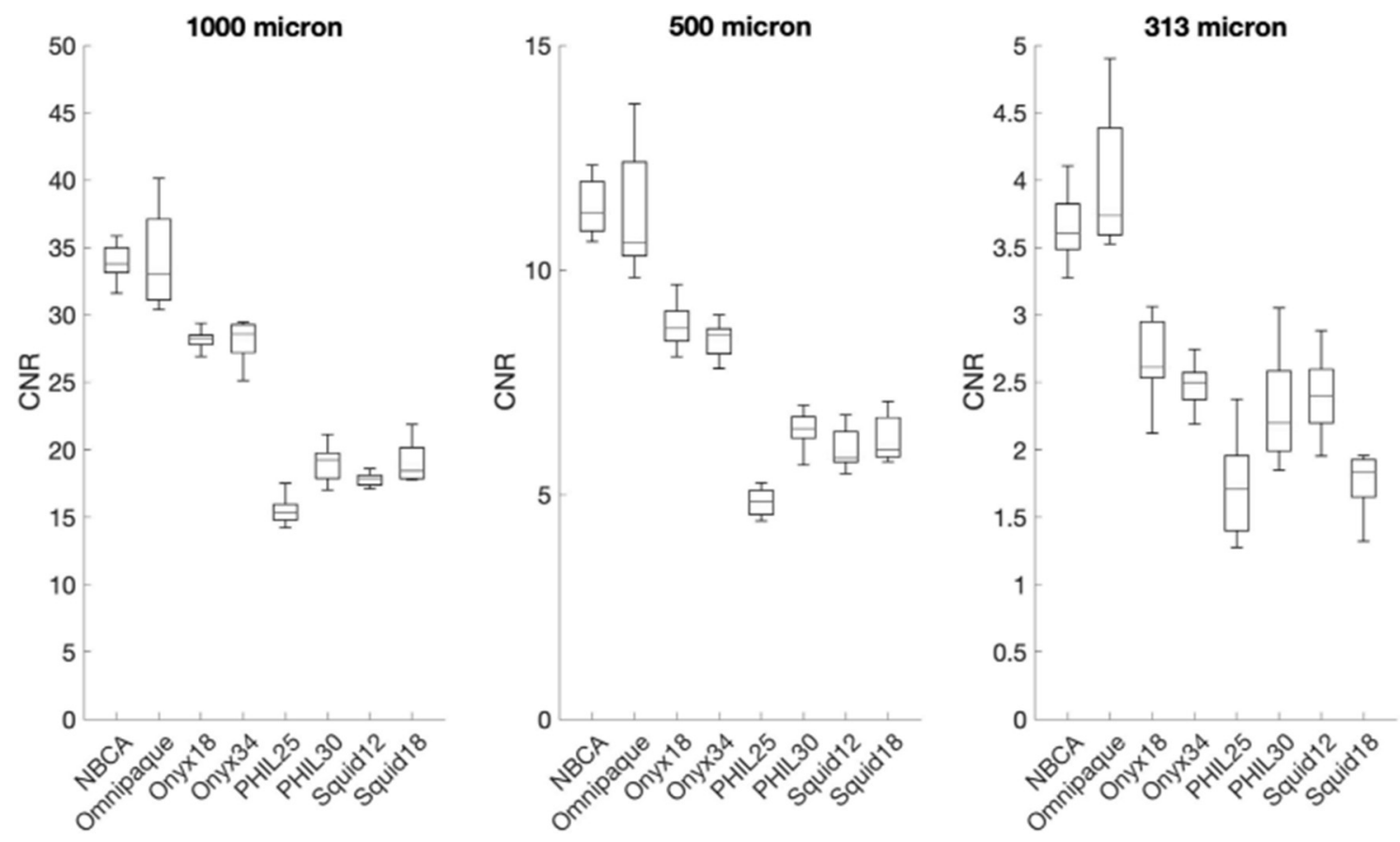

Abstract 0-015 Figure 1

(CNR) and calculated as mean peak signal minus mean background signal divided by the standard deviation of the background signal.

Results Omnipaque 300 and Trufill demonstrated significantly the highest CNR (CNR 11.3-11.4 at 500 micron and CNR $34.1-34.2$ at 1000 micron). Onyx 18 and Onyx 34 (8.5-8.8 at 500 micron and 28.1-28.2 at 1000 micron) both showed significantly higher CNR than all SQUID ${ }^{\oplus}$ and PHIL $^{\oplus}$ concentrations. There was no significant difference between SQUID ${ }^{\circledR} 12$, SQUID ${ }^{\circledast} 18$, and PHIL $^{\oplus} 30$ (CNR 6-6.4 at 500 micron and CNR 17.8-19 at 1000 micron). PHIL $^{\oplus} 25$ (CNR 4.8 at 500 micron and CNR 15.4 at 1000 micron) demonstrated the lowest CNR for all measurements. CNR results were consistent across 1000 and 500 micron tubing sizes with minor variation at 313 micron.

Conclusions There is significant variability in CR for the various LEAs under the RM conditions used in this study. Significantly different CR between most LEAs (except Trufill ${ }^{\circledR}$ ) and iodinated contrast media was evident and should be considered prior to injection. Knowledge and understanding of different visualization performance among various LEAs are important to avoid non-target vessel embolization. Subsequent studies are needed to further establish this method for improving image quality and increase patient's safety during EVT of cerebral AVMs and DAVFs.

Disclosures J. Mason: 1; C; Balt.

\section{0-016 THE DISTRIBUTION AND ROLE OF M1 AND M2 MACROPHAGES IN HEALING OF ANEURYSMS AFTER PLATINUM COIL EMBOLIZATION}

${ }^{1} Z$ Khashim*, ${ }^{1} D$ Daying, ${ }^{1} D$ Yong Hong, ${ }^{2} S$ Herting, ${ }^{1} T$ Caracena, ${ }^{1} D$ Jakaitis, ${ }^{2} D$ Maitland, 'DF Kallmes, 'R Kadirvel. 'Radiology, Mayo Clinic, Rochester, MN; ${ }^{2}$ Biomedical Engineering, Texas AandM University, College Station, TX

10.1136/neurintsurg-2019-SNIS. 16
Introduction Pro-inflammatory coatings of platinum coils have been used in the treatment of intracranial, saccular aneurysms but failed to reduce recurrence rates. Dysregulation of macrophage polarization, in which pro-inflammatory M1 macrophage population fail to transition to an anti-inflammatory M2 macrophage phenotype, is a characteristic feature of poorly healing wounds. In this study, we aimed to evaluate the distribution of M1 and M2 macrophages and to analyze their association with healing in aneurysms embolized by endovascular coiling.

Methods We created 17 elastase-induced aneurysms in female rabbits and subsequently embolized with platinum coils. Aneurysm occlusions were evaluated with angiographic imaging. Aneurysm tissue was harvested at 1, 3, and 6 months for evaluation. Quantification of M1 and M2 macrophage expression was performed by immunofluorescence. Collagen deposition was determined by Masson's trichrome staining technique. Histologic grading of aneurysms was also performed.

Results Angiographic data indicated no significant differences in the geometry of aneurysms between the three treatment-tosacrifice time groups. M1 macrophage expression in aneurysm tissues was highest at 1 month post-treatment and progressively decreased at 3- and 6 months. Expression of M2 macrophages progressively increased at 3 months and 6 months post-treatment. Collagen deposition was highest at 6 months post-treatment. Our data demonstrated a moderate to weak positive relationship between M2 macrophage expression and collagen deposition and total histologic scores and a strongly positive relationship between M2 macrophage expression and total histologic scores at 6-months post-treatment.

Conclusion Our study showed that M2 macrophage expression had a strong positive relationship with total histologic scores at a later stage of healing after endovascular coiling. These findings suggest that interventions aimed at stimulating M2 macrophage expression may improve aneurysm healing after embolization. 